\title{
Convective Models of Jupiter's Zonal Jets with Realistic and Hyper-Energetic Excitation Source
}

\author{
Hans G. Mayr ${ }^{1}$, Kwing L. Chan ${ }^{2}$ \\ ${ }^{1}$ NASA Goddard Space Flight Center, Greenbelt, USA (Retired) \\ ${ }^{2}$ State Key Laboratory for Lunar and Planetary Sciences, Macau University of Science and Technology, Macau, China \\ Email: hmayr2@verizon.net, klchan@must.edu.mo
}

How to cite this paper: Mayr, H.G. and Chan, K.L. (2019) Convective Models of Jupiter's Zonal Jets with Realistic and Hyper-Energetic Excitation Source. International Journal of Astronomy and Astrophysics, 9, 292-301.

https://doi.org/10.4236/ijaa.2019.93021

Received: July 10, 2019

Accepted: September 15, 2019

Published: September 18, 2019

Copyright $\odot 2019$ by author(s) and Scientific Research Publishing Inc. This work is licensed under the Creative Commons Attribution International License (CC BY 4.0).

http://creativecommons.org/licenses/by/4.0/

\section{Abstract}

Numerical simulations of Jupiter's zonal jets are presented, which are generated with realistic and hyper energetic source. The models are three dimensional and nonlinear, applied to a gas that is convective, stratified and compressible. Two solutions are presented, one for a shallow $0.6 \%$ envelope, the other one 5\% deep. For the shallow model (SM), Jupiter's small energy flux was applied with low kinematic viscosity. For the deep model (DM), the energy source and viscosity had to be much larger to obtain a solution with manageable computer time. Alternating zonal winds are generated of order $100 \mathrm{~m} / \mathrm{s}$, and the models reproduce the observed width of the prograde equatorial jet and adjacent retrograde jets at $20^{\circ}$ latitude. But the height variations of the zonal winds differ markedly. In SM the velocities vary radially with altitude, but in DM Taylor columns are formed. The dynamical properties of these divergent model results are discussed in light of the computed meridional wind velocities. With large planetary rotation rate $\Omega$, the zonal winds are close to geostrophic, and a quantitative measure of that property is the meridional Rossby number, $\mathrm{Ro}_{\mathrm{m}}$. In the meridional momentum balance, the ratio between inertial and Coriolis forces produces $\mathrm{Ro}_{\mathrm{m}}=\mathrm{V}^{2} / \Omega \mathrm{LU}, \mathrm{U}$ zonal, $\mathrm{V}$ meridional winds, L horizontal length scale. Our analysis shows that the meridional winds vary with the viscosity like $v^{1 / 2}$. With much larger viscosity and meridional winds, the Rossby number for DM is much larger, $\mathrm{Ro}_{\mathrm{m}}(\mathrm{DM})>>\mathrm{Ro}_{\mathrm{m}}(\mathrm{SM})$. Compared to the shallow model with zonal winds varying radially, the deeper and more viscous model with Taylor columns is much less geostrophic. The zonal winds of numerical models in the literature tend to be independent of the energy source, in agreement with the present results. With $10^{4}$ times larger energy flux, the zonal winds for DM only increase by a factor of 3 , and the answer is provided by the zonal momentum budget with meridional winds, $\mathrm{VU} / \mathrm{L}=\Omega \mathrm{V}$, yielding $\mathrm{U}=\Omega \mathrm{L}$, independent of the source. The same relationship produces the zonal Rossby number, $\mathrm{Ro}_{\mathrm{z}}=$ 
$\mathrm{U} / \Omega \mathrm{L}$, of Order 1 , which is commonly used as a dimensionless measure of the zonal wind velocities.

\section{Keywords}

Convective Atmosphere, Zonal-Mean Variations, Radial Zonal Winds, Taylor Column Zonal Winds, Energy Invariantzonal Winds, Geostrophic Balance, Meridional Wind Dynamics

\section{Introduction}

The alternating wind bands observed on Jupiter (e.g., Smith et al. [1] [2]; Porco et al. [3]) have been simulated with numerical models that can be placed in two groups. One is confined to the stable region in the clouds and accounts for horizontal cascading of turbulence (e.g., Rhines [4]; Marcus et al. [5]; Showman et al. [6]). The other class of models extends below the cloud top and accounts for convective energy transport from the interior (e.g., Busse [7]; Mayr et al. [8]; Sun et al. [9]; Ingersoll et al. [10]; Zhang and Schubert [11]; Christensen [12]; Aurnou and Olson [13]; Heimpel and Aurnou [14]; Chan and Mayr [15] [16]; Cai and Chan [17]).

In this paper, we present simulations of Jupiter's alternating wind bands generated by convection. The numerical models are nonlinear and three dimensional, applied to a gas that is stratified and compressible. Two solutions are discussed, one for a shallow envelope $0.6 \%$ of planetary radius, the other one $5 \%$ deep more commensurate with reality. For the shallow model (SM), the small planetary energy flux from the interior was applied together with the corresponding low kinematic viscosity. For the deep model (DM), the applied energy and viscosity had to be much larger to achieve sufficient fast thermal relaxation with manageable computer time, conceptually similar to the deep convective models that have appeared in print.

The mean zonal winds generated with SM and DM differ markedly, varying radially with altitude and aligned along Taylor columns, respectively, and they are discussed in light of the computed meridional winds.

\section{Numerical Models}

The numerical models discussed are based on a series of earlier studies (Chan and Sofia [18] [19]; Chan et al. [20]; Chan [21] [22]; Chan and Mayr [15]). Jupiter's atmosphere is treated as an ideal gas, and a uniform energy flux is applied at the bottom. The energy is carried by convection over $95 \%$ of the layer, and it is emitted by radiative diffusion from a thin stable layer at the top. The vertical component of the Coriolis force is accounted for, which figures prominently in the momentum budget. A uniform kinematic viscosity is adopted to dissipate the kinetic energy generated by convection, with the Prandtl number set to $1 / 3$. 
Stress-free and impenetrable boundary conditions are applied at the top and bottom.

\subsection{Shallow Model (SM)}

The numerical code employs the transformed spectral procedure with associated vector spherical harmonics and solves the time-dependent 3D nonlinear Navier Stokes equations (Chan et al. [20]; Chan and Mayr [15]). The solution procedure is carried out in two stages. For each time step, the linearized equations are solved implicitly in spectral space, and the nonlinear terms are then computed explicitly in physical space.

The shallow model (SM) extends into the convection region with $\Delta \mathrm{r}=0.6 \%$ depth of the planetary radius. Jupiter's energy flux, $\mathrm{F}=5.4 \mathrm{~W} / \mathrm{m}^{2}$, is applied at the bottom boundary, but the smaller Solar input is ignored. The kinematic viscosity, $v=5.6 \mathrm{~m}^{2} / \mathrm{s}$, is employed, which produces the dimensionless Ekman number, $\mathrm{E}=v / \Omega \Delta \mathrm{r}^{2}=1.7 \times 10^{-7}$, with planetary rotation rate, $\Omega=1.778 \times 10^{-4}$ $\mathrm{rad} / \mathrm{s}$ (9.8 hours). SM applies triangular truncation of spherical harmonics up to degree 20 (T20) with limited latitudinal resolution, and 68 radial grid levels for the $0.6 \%(430 \mathrm{~km})$ shell. With low kinematic viscosity and small Ekman number, the model ran 1 year to reach thermal relaxation, and we present in the following the time average zonal mean variations of the computed zonal wind velocities.

Figure 1 is a composite of the zonal wind properties that characterize the SM simulation of Jupiter's alternating wind bands. Except for the color code, Figure 1 (b) is taken from Chan and Mayr [15] and shows the winds at 4 different altitude levels, identified in the underlying scale of the fractional radius. In qualitative agreement with observations, alternating wind bands are generated. A dominant prograde equatorial jet is produced with velocities close to $70 \mathrm{~m} / \mathrm{s}$, which is within a factor of 2 of the observed values. Adjacent to the equatorial jet, strong retrograde winds are generated at $20^{\circ}$ latitudes, in agreement with the Voyager observations (Smith et al. [2]). But the alternating jets at higher latitudes are too wide and too few in number.

The Taylor-Proudman theorem predicts that for a fluid that is geostrophic and incompressible, the zonal winds become aligned along Taylor columns (TC), and this is the prevailing picture of convective models of the Jupiter atmosphere (e.g., Christensen [12]; Aurnou and Olson [13]; Heimpel and Aurnou [14]). In typical TC models, the tangent cylinder to the inner boundaries determines the width of the equatorial jet, which is defined by the location of the maximum retrograde zonal winds. As illustrated in Figure 1(a), the angular span for the intersections of the tangent cylinder extends to $6^{\circ}$ latitudes. In contrast, the equatorial jet in Figure 1(b) has a much larger $20^{\circ}$ span, which demonstrates that SM does not produce a TC pattern at low latitudes. Figure 1(b) shows that the zonal winds at different levels vary with altitude. This picture is brought into focus in Figure 1(c), where the wind pattern of the southern hemisphere is displayed along an expanded altitude scale. 
Shallow Model (SM)

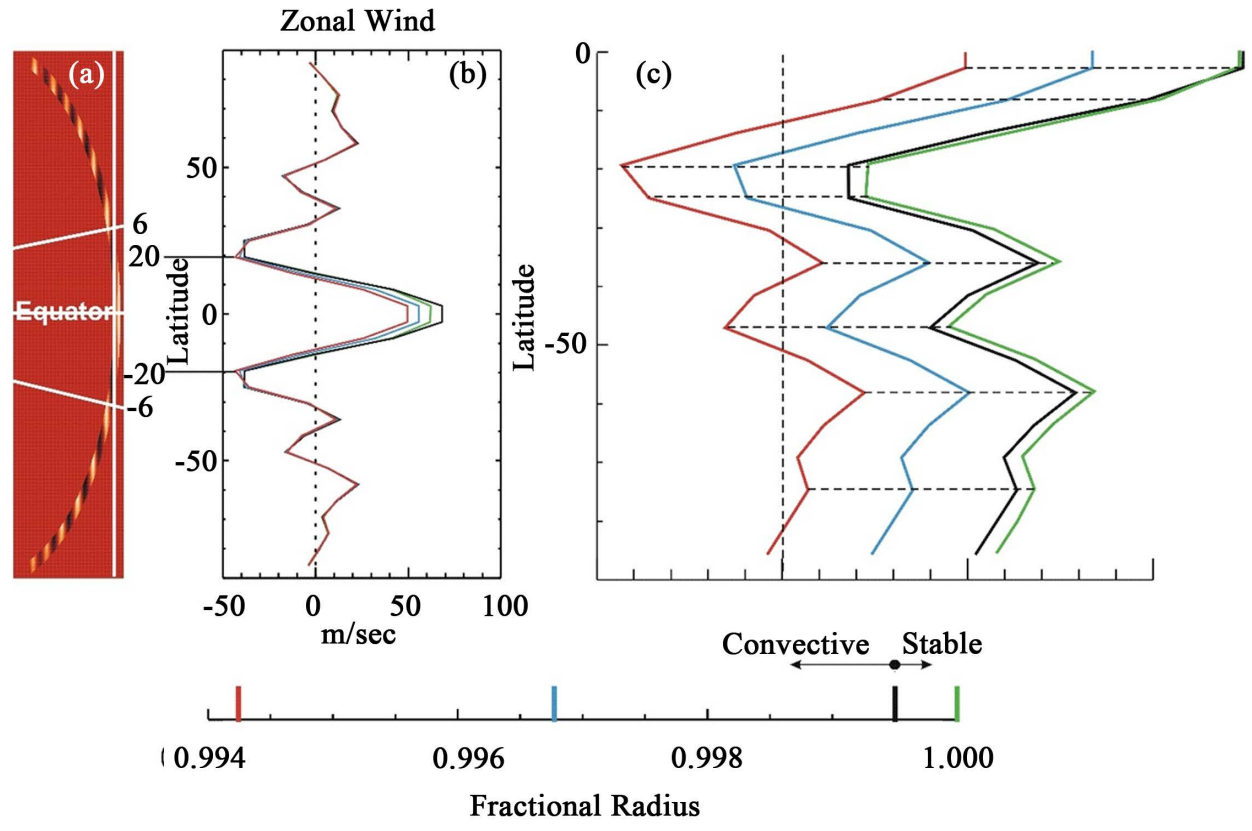

Figure 1. Composite of the zonal wind properties that characterize the shallow model (SM) simulation of Jupiter's alternating wind bands. Except for the color code, (b) is taken from Chan and Mayr [15] and shows the winds at 4 different altitude levels, identified in the underlying scale of the fractional radius. As illustrated in (a), the angular span for the intersections of the tangent cylinder extends to $6^{\circ}$ latitudes. In contrast, the equatorial jet in (b) has a much larger $20^{\circ}$ span, which demonstrates that SM does not produce a Taylor column pattern parallel to the rotation axis. This picture is brought into focus in (c), where the zonal wind pattern of the southern hemisphere is displayed on an expanded altitude scale.

The zonal winds of SM are not aligned along the rotation axis but vary radially with altitude, and the question is whether this property will survive in deeper models with realistic planetary parameters and sufficient low viscosity. Short of the results from such a computationally demanding study, it is instructive to examine a simulation from a numerical model, stratified and compressible, which is much deeper but employs a much larger energy source and viscosity.

\subsection{Deep Model (DM)}

Considering Ohmic dissipation associated with Jupiter's magnetic field and measured conductivity, Liu et al. [23] estimated that the zonal winds cannot penetrate below 0.96 radius. This is on the order of depth employed in present convective models (e.g., Christensen [12]; Aurnou and Olson [13]; Heimpel and Aurnou [14]), referred to as $\mathrm{CAOH}$. $\mathrm{CAOH}$ simulate the prograde equatorial jet and alternating wind bands with large viscosity, applying much larger energy flux than Jupiter's.

Following $\mathrm{CAOH}$, a deep model (DM) was constructed with relative depth $\Delta \mathrm{r}=5 \%$, which was presented by Chan and Mayr [16]. For this model, a grid point formulation was employed to integrate the time-dependent $3 \mathrm{D}$ nonlinear 
Navier Stokes equations across the globe (Chan [22]). The model was run with an energy flux, $\mathrm{F}=7.8 \times 10^{4} \mathrm{~W} / \mathrm{m}^{2}$, a factor of $1.4 \times 10^{4}$ larger than that of Jupiter. For the kinematic viscosity the value $v=2.3 \times 10^{5} \mathrm{~m}^{2} / \mathrm{s}$ was chosen, which produces the Ekman number $\mathrm{E}=v / \Omega \Delta \mathrm{r}^{2}=1.0 \times 10^{-4}$. With this large viscosity, the model ran 2 months to produce the numerical results.

Analogous to Figure 1, we present in Figure 2 the zonal winds from the deep model (DM). In agreement with observations, multiple alternating jets are generated that extend to high latitudes. The prograde equatorial jet has a velocity of about $200 \mathrm{~m} / \mathrm{s}$, and the adjacent retrograde jets are $20^{\circ}$ wide in agreement with Voyager observations. But unlike the latitudinal variations of the computed zonal winds, on Jupiter the equatorial jet dominates.

In contrast to SM, the zonal winds from DM clearly show the pattern of Taylor columns. Illustrated in Figure 2(a), the angular span for the intersecting tangent cylinder is $\pm 20^{\circ}$ wide, determined by the $5 \%$ depth of the model. This agrees with the latitudes of the retrograde jets in Figure 2(b), which define the width of the equatorial wind pattern. Around the equator, the winds at different altitude levels vary between -150 and $+150 \mathrm{~m} / \mathrm{s}$, aligned parallel to the tangent cylinder. Away from the equator, the deeper zonal winds (positive or negative) occupy lower latitudes, brought into focus in Figure 2(c), which is consistent with alignment along the rotation axis. The zonal winds of DM form Taylor columns, in substantial agreement with the convection models of $\mathrm{CAOH}$.

Deep Model (DM)

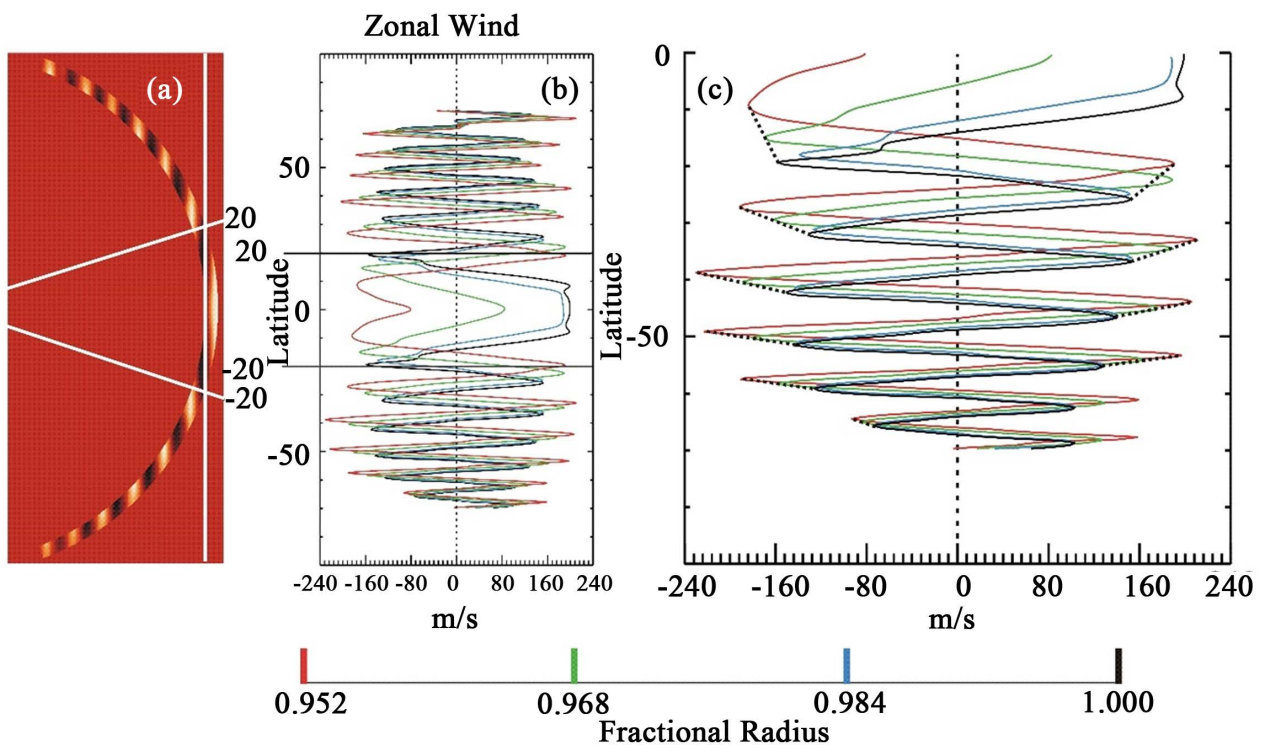

Figure 2. Composite of the zonal wind properties, similar to Figure 1, but for the deep model (DM). Figure 1(b) is taken from Chan and Mayr [16] and shows the winds at 4 different altitude levels, identified in the underlying scale of the fractional radius. (a) shows that the angular span of the tangent cylinder extends to $20^{\circ}$ latitudes, in agreement with the width of the equatorial jet in (b). The zonal winds form a Taylor column pattern, brought into focus in (c). 


\section{Discussion}

For model simulations of a convective Jovian atmosphere that is both stratified and compressible, it is remarkable that the resulting alternating wind bands are formed with such different altitude patterns. In the shallow model the zonal velocities vary radially, but in the deeper model the variations are aligned along the rotation axis to form Taylor columns. Apart from the different vertical domains, the applied energy source and related viscosity must come into play.

The Taylor-Proudman theorem applies if the zonal winds are in geostrophic balance. For the shallow model (SH), geostrophy was explicitly demonstrated by comparing the meridional pressure gradient with the Coriolis force (Chan and Mayr [15]). A quantitative measure of geostrophic balance is the meridional Rossby number, $\mathrm{Ro}_{\mathrm{m}}$, which is defined as the ratio between inertial and Coriolis forces. Among the nonlinear inertial accelerations in the meridional momentum equation that describes the mean zonal wind, $\mathrm{U}$, the term $\mathrm{V} \partial \mathrm{V} / \partial \theta$ is the largest, $\theta$ latitude and $\mathrm{V}$ mean horizontal meridional wind. Compared with the Coriolis force term $\Omega U$, the meridional Rossby number then can be estimated, $\mathrm{Ro}_{\mathrm{m}}=$ $\mathrm{V}^{2} / \mathrm{L} \Omega \mathrm{U}$, with the characteristic horizontal length scale, $\mathrm{L}=\lambda / 2 \pi$ ( $\lambda$, horizontal wavelength) that is related to the planetary radius, $r$.

Figure 3 shows the meridional winds, on the left for the shallow model (SM), on the right for the deep model (DM). At any given latitude, the converging and diverging velocities have opposite directions in the northern and southern hemispheres. Apart from that, the different wind patterns mirror those of the zonal velocities (Figure 1, Figure 2): a dominant single cell circulation for SM with dominant equatorial jet, and a multi-cellular circulation for the alternating wind bands of DM. Obeying flow continuity, the meridional velocities are much larger
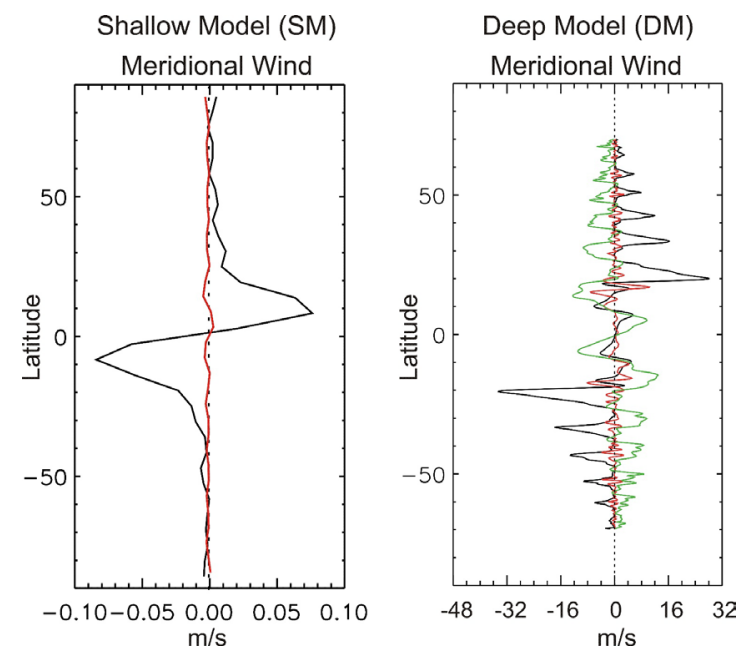

Figure 3. Computed meridional winds, left for the shallow model (SM), right the deep model (DM). Converging and diverging velocities have opposite directions in the northern and southern hemispheres. Obeying flow continuity, the meridional velocities are much larger at the top of the domain (black versus red/green), where the ambient densities are much smaller (identical in both models). Note that the maximum wind velocities for SM are very small less than $0.10 \mathrm{~m} / \mathrm{s}$, in contrast to DM with velocities close to $30 \mathrm{~m} / \mathrm{s}$ (figures are taken from Chan and Mayr [16]). 
at the top of the domain (black versus green/red), where the ambient densities are much smaller (identical in both models). Apart from the contrasting wind patterns, the meridional winds feature large differences in magnitude. For SM at the top of the domain, the maximum wind velocities are very small less than 0.10 $\mathrm{m} / \mathrm{s}$, in contrast to DM with velocities close to $30 \mathrm{~m} / \mathrm{s}$.

Given the wind velocities, the corresponding model parameters are listed in Table 1 for $\mathrm{L}=\mathrm{r}$. The applied viscosities, $v$, are sufficiently small in both models to assure that rotation dominates and the Ekman numbers are very small, $\mathrm{E}<<$ 1. This holds also for the deep model with much larger $v$. The meridional Rossby numbers are very small as well, $\mathrm{Ro}_{\mathrm{m}}<<1$, demonstrating that both model results are approximately in geostrophic balance. But $\mathrm{Ro}_{\mathrm{m}}$ is much larger for $\mathrm{DM}$ with Taylor columns.

Our model results reveal an intriguing relationship between the viscosity and meridional winds. As shown in Table 1, last column, the dimensionless numbers for the two models, $\mathrm{V}^{2} / \nu \Omega$ differ only by a factor of two, which is remarkable considering that the input parameters for the energy source and viscosity differ by orders of magnitude. The chosen viscosity apparently determines the magnitude of the meridional wind, $\mathrm{V}$ varying with $(\nu \Omega)^{1 / 2}$.

Another intriguing property of the numerical results is the invariance of the zonal velocities in relation to the energy source. As shown in Table 1 , the winds increase from $70 \mathrm{~m} / \mathrm{s}(\mathrm{SM})$ to $200 \mathrm{~m} / \mathrm{s}(\mathrm{DM})$, produced by an energy flux a factor of $1.4 \times 10^{4}$ larger. This trend is observed in planetary atmospheres. And the Jupiter models in the literature, with energies far exceeding the planetary value, all feature zonal wind velocities comparable to those observed.

Mayr et al. [24] addressed this problem with a simplified 2D scale analysis of the zonal momentum budget, where the Coriolis force, $\Omega \mathrm{V}$, is balanced by the viscous stress of the zonal winds, $\mathrm{UK} / \mathrm{L}^{2}$, with $\mathrm{K}$ the eddy viscosity and $\mathrm{L}$ the horizontal scale of the circulation. Applying mixing length theory, $\mathrm{K}=\mathrm{VL}$, one obtains $\mathrm{U}=$ $\Omega \mathrm{L}$, which produces for the Jovian circulation zonal winds of order $100 \mathrm{~m} / \mathrm{s}$.

Essentially, the solution is provided by the nonlinear zonal momentum budg-

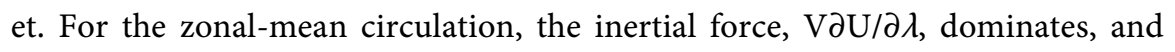
the balance with the Coriolis force yields, $\mathrm{VU} / \mathrm{L}=\Omega \mathrm{V}$, to produce $\mathrm{U}=\Omega \mathrm{L}$, independent of the energy source. The same relationship produces the zonal Rossby number, $\mathrm{Ro}_{\mathrm{z}}=\mathrm{U} / \Omega \mathrm{L}$, of order 1 , which is commonly used as dimensionless measure of the zonal wind velocities.

Table 1. The chosen model parameters for SM and DM are listed: $\Delta \mathrm{r}(\mathrm{m})$ depth of convection region, $\mathrm{F}\left(\mathrm{W} / \mathrm{m}^{2}\right)$ planetary energy flux from interior, and $v$ kinematic viscosity. The model generated zonal and meridional wind velocities, $\mathrm{U}(\mathrm{m} / \mathrm{s}), \mathrm{V}(\mathrm{m} / \mathrm{s})$, are shown for the top of the atmosphere. The Ekman number, $\mathrm{E}=v / \Omega \Delta \mathrm{r}^{2}$, is a measure of the importance of planetary rotation. And the meridional Rossby number, $\mathrm{Ro}_{\mathrm{m}}=\mathrm{V}^{2} / \mathrm{r} \Omega \mathrm{U}$, is a measure of geostrophic balance for the zonal winds. The model invariant ratios, $\mathrm{V}^{2} / \chi \Omega$, indicate that the meridional winds, $\mathrm{V}$ vary with $(\nu \Omega)^{1 / 2}$.

\begin{tabular}{ccccccccc}
\hline & $\Delta \mathrm{r}(\mathrm{m})$ & $\mathrm{F}\left(\mathrm{W} / \mathrm{m}^{2}\right)$ & $v\left(\mathrm{~m}^{2} / \mathrm{s}\right)$ & $\mathrm{U}(\mathrm{m} / \mathrm{s})$ & $\mathrm{V}(\mathrm{m} / \mathrm{s})$ & $\mathrm{E}=v / \Omega \Delta \mathrm{r}^{2}$ & $\mathrm{Ro}_{\mathrm{m}}=\mathrm{V}^{2} / \mathrm{r} \Omega \mathrm{U}$ & $\mathrm{V}^{2} / \nu \Omega$ \\
\hline Shallow SM & $4.3 \times 10^{5}$ & 5.4 & 5.6 & 70 & 0.1 & $1.7 \times 10^{-7}$ & $1.1 \times 10^{-8}$ & 10.0 \\
Deep DM & $3.6 \times 10^{6}$ & $7.5 \times 10^{4}$ & $2.3 \times 10^{5}$ & 200 & 30 & $1.0 \times 10^{-4}$ & $3.4 \times 10^{-4}$ & 22.0 \\
\hline
\end{tabular}


In geostrophic balance, the zonal winds are produced by latitudinal temperature/pressure variations. But the source that produces the temperature variations is also generating the meridional circulation that redistributes or dissipates the kinetic energy. The zonal velocities thus tend to be independent of the energy source.

\section{Summary}

Numerical simulations of Jupiter's zonal jets are discussed, which are generated with models that are three dimensional and fully nonlinear, applied to a gas that is convective, stratified and compressible. Solutions are presented for shallow and deep atmospheric envelopes, generated with realistic and hyper-energetic source. In the shallow model (SM) with realistic energy source, the zonal winds vary radially with altitude, in contrast to the energetic deep model (DM) where the winds are aligned along the rotation axis to form Taylor columns (TC). In agreement with observations, both models produce prograde equatorial jets of order $100 \mathrm{~m} / \mathrm{s}$. Both models also reproduce the observed width of the equatorial jet with adjacent retrograde jets at $20^{\circ}$ latitude-a natural outcome for SM, but determined by the chosen $5 \%$ depth of DM with TC.

The dynamical properties of these divergent model results are discussed in light of the meridional winds, which are small in magnitude compared with the zonal winds. But unlike the rotational zonal winds, the meridional winds have divergence, and thus are involved with energy and momentum transport, which is of central importance for understanding the zonal mean circulation.

The Rossby number, $\mathrm{Ro}_{\mathrm{m}}$, for the meridional momentum balance is the quantitative measure of geostrophy, and it is a quadratic function of the meridional winds. For DM with large viscosity and TC, the meridional winds and $\mathrm{Ro}_{\mathrm{m}}$ are orders of magnitude larger compared to SM. DM is much less geostrophic. Ranking geostrophy cannot explain the difference between SM and DM. For Taylor columns to form, Taylor-Proudman requires that the gas is also incompressible, in addition to geostrophic. But both models treat the atmosphere as compressible. In models like DM with large viscosity, the enhanced energy transport by the meridional winds has the capacity to reduce the vertical variations in the latitudinal temperature distribution to produce a barotropic environment that favors the formation of TC. The question is whether deeper models, with realistic energy flux and low viscosity, will produce zonal winds that vary radially with altitude like SM.

The numerical results presented highlight an important property of planetary atmospheres, the invariance of the zonal winds in relation to the energy source. With $10^{4}$ times larger source, the velocities of DM increase only by a factor of 3 . And the Jupiter models in the literature with energies far exceeding the planetary value all feature zonal winds comparable to those observed. Following up on an earlier paper (Mayr et al. [24]), the solution of this problem is provided by the nonlinear zonal momentum budget with meridional winds. It produces zonal winds varying with the planetary rotation rate and horizontal scale of the circu- 
lation, independent of the energy source.

\section{Acknowledgements}

Funded by the Science and Technology Development Fund, Macau SAR (File No. 0045/2018AFJ). The reviewer's comments contributed significantly to improve the presentation of the paper. This work was supported by the State Key Laboratory for Lunar and Planetary Sciences, Macau University of Science and Technology.

\section{Conflicts of Interest}

The authors declare no conflicts of interest regarding the publication of this paper.

\section{References}

[1] Smith, B.A., Soderblom, L.A., Johnson, T.V., et al. (1979a) The Jupiter System through the Eyes of Voyager 1. Science, 204, 951-972.

https://doi.org/10.1126/science.204.4396.951

[2] Smith, B.A., Soderblom, L.A., Beeebe, R., et al. (1979b) The Galilean Satellites and Jupiter: Voyager 2 Imaging Satellite Results. Science, 206, 927-950. https://doi.org/10.1126/science.206.4421.927

[3] Porco, C.C., West, R.A., McEwen, A., et al. (2003) Cassini Imaging of Jupiter's Atmosphere, Satellites, and Rings. Science, 299, 1541-1547.

https://doi.org/10.1126/science.1079462

[4] Rhines, P.B. (1975) Waves and Turbulence on a Beta-Plane. Journal of Fluid Mechanics, 69, 417-443. https://doi.org/10.1017/S0022112075001504

[5] Marcus, P.S., Kundu, T. and Lee, C. (2000) Vortex Dynamics and Zonal Flows. Physics of Plasmas, 7, 1630-1640. https://doi.org/10.1063/1.874045

[6] Showman, A.P., Gierasch, P.J. and Lian, Y. (2006) Deep Zonal Winds Can Result from Shallow Driving in a Giant-Planet Atmosphere. Icarus, 182, 513-526. https://doi.org/10.1016/j.icarus.2006.01.019

[7] Busse, F.H. (1976) Simple Model of Convection in the Jovian Atmosphere. Icarus, 29, 255-260. https://doi.org/10.1016/0019-1035(76)90053-1

[8] Mayr, H.G., Harris, I. and Chan, K.L. (1984) Differential Rotation in a Solar-Driven Quasi-Axisymmetric Circulation. Earth Moon Planets, 30, 245-274. https://doi.org/10.1007/BF00056202

[9] Sun, Z.P., Schubert, G. and Glatzmaier, G.A. (1993) Banded Surface Flow Maintained by Convection in a Model of the Rapidly Rotating Giant Planets. Science, 260, 661-664. https://doi.org/10.1126/science.260.5108.661

[10] Ingersoll, A.P., Gierasch, P.J., Banfield, D., et al. (2000) Moist Convection as an Energy Source for the Large-Scale Motions in Jupiter's Atmosphere. Nature, 403, 630-632. https://doi.org/10.1038/35001021

[11] Zhang, K. and Schubert, G. (2000) Tele-Convection: Remotely Driven Thermal Convection in Rotating Stratified Spherical Layers. Science, 290, 1944. https://doi.org/10.1126/science.290.5498.1944

[12] Christensen, U.R. (2001) Zonal Flow Driven by Deep Convection in the Major Planets. Geophysical Research Letters, 28, 2553-2556. 
https://doi.org/10.1029/2000GL012643

[13] Aurnou, J.M. and Olson, P.I. (2001) Strong Zonal Winds from Thermal Convection in a Rotating Spherical Shell. Geophysical Research Letters, 28, 2557-2559. https://doi.org/10.1029/2000GL012474

[14] Heimpel, M. and Aurnou, J. (2007) Turbulent Convection in a Rapidly Rotating Spherical Shell: A Model for Equatorial and High Latitude Jets on Jupiter and Saturn. Icarus, 187, 540-557. https://doi.org/10.1016/j.icarus.2006.10.023

[15] Chan, K.L. and Mayr, H.G. (2008) A Shallow Convective Model for Jupiter's Alternating Wind Bands. Journal of Geophysical Research, 113, E10002. https://doi.org/10.1029/2008JE003124

[16] Chan, K.L. and Mayr, H.G. (2008) Convective Models of Jupiter's Wind Bands: Transition from Deep to Shallow Envelopes. 40 th Annual Meeting of Division of Planetary Sciences of American Astronomical Society, Ithaca.

[17] Cai, T. and Chan, K.L. (2012) Three-Dimensional Numerical Simulation of Convection in Giant Planets: Effects of Solid Core Size. Planetary and Space Science, 71, 125-130. https://doi.org/10.1016/j.pss.2012.07.023

[18] Chan, K.L. and Sofia, S. (1986) Turbulent Compressible Convection in a Deep Atmosphere: III Tests on the Validity and Limitation of the Numerical Approach. The Astrophysical Journal, 307, 222-241. https://doi.org/10.1086/164409

[19] Chan, K.L. and Sofia, S. (1989) Turbulent Compressible Convection in a Deep Atmosphere: IV Results of Three-Dimensional Computations. The Astrophysical Journal, 336, 1022-1040. https://doi.org/10.1086/167072

[20] Chan, K.L., Mayr, H.G., Mengel, J.G. and Harris, I. (1994) A "Stratified" Spectral Model for Stable and Convective Atmospheres. Journal of Computational Physics, 113, 165-176. https://doi.org/10.1006/jcph.1994.1128

[21] Chan, K.L. (2001) Rotating Convection in f-Planes: Mean Flow and Reynolds Stress. The Astrophysical Journal, 548, 1102-1117. https://doi.org/10.1086/318989

[22] Chan, K.L. (2006) A Finite-Difference Convective Model for Jupiter's Equatorial Jet. In: Kupka, F., et al., Eds., Proceedings IAU Symposium No. 239: Convection in Astrophysics, Cambridge University Press, New York, 230-232. https://doi.org/10.1017/S174392130700049X

[23] Liu, J., Goldreich, M. and Stevens, D.J. (2008) Constraints on Deep-Seated Zonal Winds Inside Jupiter and Saturn. Icarus, 196, 653-664. https://doi.org/10.1016/j.icarus.2007.11.036

[24] Mayr, H.G., Chan, K.L., Harris, I. and Schatten, K. (1991) What Maintains the Zonal Circulation in Planetary Atmospheres. The Astrophysical Journal, 367, 361-366. https://doi.org/10.1086/169634 